\title{
Avaliação e Reabilitação Neuropsicológica após Lesão Encefálica Adquirida
}

\author{
Paula Adriana Rodrigues de Gouveia ${ }^{1}$ \\ Shirley Silva Lacerda \\ Ana Merzel Kernkraut \\ Hospital Israelita Albert Einstein, São Paulo, SP, Brasil
}

\begin{abstract}
Resumo
Sintomas de desconexão inter-hemisférica após lesão cerebral adquirida são pouco usuais na prática clínica atualmente. Os sintomas costumam diminuir espontaneamente e podem incluir anomia táctil, agrafia à esquerda e sinais de competição entre os hemisférios, como o "sinal da mão alienígena". Este trabalho tem por objetivo apresentar um relato de caso clínico de paciente de 33 anos, que evoluiu com sintomas de desconexão inter-hemisférica e alterações de funções executivas, após intervenção cirúrgica para remoção de tumor em região da hipófise, além de avaliar a aplicabilidade de programa de reabilitação neuropsicológica para as queixas cognitivas apresentadas. O paciente foi avaliado com instrumentos neuropsicológicos padronizados antes de iniciar a reabilitação e próximo à alta do tratamento. $\mathrm{O}$ estudo de caso foi realizado a partir da análise de dados de intervenção e evolução clínica do paciente em prontuário institucional (resultados de exame de avaliação neuropsicológica e registros de intervenções em sessões de reabilitação). Ao longo de sua evolução, o paciente apresentou melhora dos sintomas cognitivos e demonstrou benefício com os treinos e estratégias utilizados para sua adaptação, bem como para retomar o trabalho e outras atividades prévias. Destaca-se ainda a importância de se realizar mais estudos de intervenções neuropsicológicas para indivíduos com déficits cognitivos.
\end{abstract}

Palavras-chave: Cognição, reabilitação, lesão, apraxia, lobo frontal.

\section{Neuropsychological Evaluation and Rehabilitation after Acquired Brain Injury}

\begin{abstract}
Symptoms of interhemispheric disconnection after acquired brain injury are unusual in the current clinical practice. Symptoms usually diminish spontaneously and may include tactile anomia, agraphia and left signs of competition between the hemispheres, such as "alien hand sign". This work presents a case report of a 33 year old patient who developed symptoms of interhemispheric disconnection and executive function deficits after surgery for removal of a tumor in the pituitary region, as well as the evaluation of the applicability of the neuropsychological rehabilitation program for the cognitive complaints presented. The patient was evaluated with standardized neuropsychological instruments before starting rehabilitation and again close to the discharge from treatment. The case study was conducted
\end{abstract}

Endereço para correspondência: Hospital Israelita Albert Einstein, Centro de Reabilitação, Av. Albert Einstein, 627, São Paulo, SP, Brasil 05652-900. E-mail: paularg@einstein.br

Agradecimentos: Ao Dr. Cícero José Nunes Vaz (Médico Fisiatra) e aos demais profissionais do Centro de Reabilitação que acompanharam o paciente D.T. (equipes da Fisioterapia, Terapia Ocupacional e da Fonoaudiologia), com quem tivemos a oportunidade de discutir o caso e trocar impressões valiosas para o planejamento do trabalho e integração da assistência ao paciente. 
by analyzing intervention data and the clinical outcome of the patient in the institutional records (results of neuropsychological evaluation examination and records of interventions in rehabilitation sessions). Throughout his evolution, the patient showed improvement in cognitive symptoms and demonstrated benefits from the training and strategies used for his adaptation and return to work and other previous activities. The study highlights the importance of conducting more studies of neuropsychological interventions for individuals with cognitive deficits.

Keywords: Cognition, rehabilitation, injury, apraxia, frontal lobe.

\section{Evaluación y Rehabilitación Neuropsicológica después de una Lesión Cerebral Adquirida}

\section{Resumen}

Los síntomas de desconexión interhemisférica después de una lesión cerebral adquirida son inusuales en la práctica clínica actual. Los síntomas generalmente disminuyen de forma espontánea y pueden incluir anomia táctil, agrafía izquierda y signos de la competencia entre los hemisférios. Este estudio tiene como objetivo presentar un caso clínico de un paciente de 33 años, que desarrollaron síntomas de desconexión interhemisférica después de la cirugía para la extirpación del tumor en la región pituitaria y alteraciones en la función ejecutiva y evaluar la aplicabilidad del programa de rehabilitación neuropsicológica para las quejas cognitivas. El paciente fue evaluado con instrumentos neuropsicológicas estandarizadas antes de comenzar la rehabilitación y cerca del máximo tratamiento. El estudio de caso se llevó a cabo a partir del análisis de datos de intervención y el desarrollo clínico del paciente registro médico institucional (resultados de los exámenes de evaluación neuropsicológica y las intervenciones en los registros de las sesiones de rehabilitación). A lo largo de su evolución, el paciente mostró una mejoría en los síntomas cognitivos y demostró un beneficio con la formación y las estrategias utilizadas para adaptarse y para reanudar el trabajo y otras actividades anteriores. También pone de relieve la importancia de llevar a cabo más estudios de intervenciones neuropsicológicas para las personas con déficits cognitivos.

Palabras clave: La cognición, la rehabilitación, lesiones, apraxia, lóbulo frontal.

Pacientes com lesões nas conexões entre os hemisférios podem apresentar sintomas característicos da síndrome de desconexão inter-hemisférica (Geschwind, 1965a; Gil, 2002; Loring, 1999; Sperry, Gazzaniga, \& Bogen, 1969). O conhecimento sobre os sintomas neuropsicológicos, decorrentes da desconexão dos hemisférios foi derivado do estudo de pacientes que tiveram ressecção do corpo caloso para tratamento de epilepsia refratária.

A síndrome de desconexão inter-hemisférica, conforme descrita por Sperry et al. (1969), caracteriza-se por sintomas como anomia táctil e visual, ausência de transferência inter-hemisférica de estimulação sensorial unilateral de ambas as mãos, hemialexia e apraxia unilateral esquerda. Os sinais de desconexão calosa não costumam ser muito óbvios e podem diminuir espontaneamente algum tempo após a lesão, além de serem compensados pelos pacientes, especialmente no caso da comissurotomia ser apenas parcial, comprometendo apenas algumas modalidades sensoriais. Eles só se tornam claros em condições específicas de exame, em que o acesso à informação ao outro hemisfério pode ser controlada (Browndyke, 2002; Rubens, Geschwind, Mahowald, \& Mastri, 1977). Sintomas como anomia táctil à esquerda (o sujeito não é capaz de nomear objetos na mão esquerda de olhos fechados, pois a informação táctil não chega ao hemisfério esquerdo para ser nomeada), agrafia esquerda (incapacidade de produzir escrita legível pelo prejuízo da transferência de informações viso cinestésicas entre os hemisférios) e sinais de conflito ou competição entre membros ou "sinal da mão alienígena" (o pa- 
ciente demonstra estranhamento frente às ações realizadas por uma das mãos ou apresenta clara competição em que uma mão desfaz o que a outra acabou de fazer), são alguns dos exemplos de manifestações clínicas desta condição (Browndyke, 2002; Geschwind, 1965b).

A avaliação neuropsicológica permite o exame detalhado de diversas esferas cognitivas, através de instrumentos específicos para cada habilidade que se pretende avaliar, permitindo a observação de sintomas associados ao quadro de desconexão inter-hemisférica. O impacto destes sintomas e outros associados à lesão encefálica, pode interferir na autonomia do paciente e no seu retorno pleno às atividades prévias. Alterações de funções executivas são exemplos de sintomas com impacto relevante na autonomia dos indivíduos afetados, pela sua repercussão na organização, planejamento e monitoramento do comportamento e das ações intencionais (Levine et al., 2011).

O tratamento de reabilitação neuropsicológica tem o objetivo de minimizar as alterações cognitivas, para que o paciente atinja seu melhor nível de funcionalidade possível na vida diária (Wilson, 2008). O trabalho é feito através de orientação sobre o funcionamento cognitivo, treinos para melhorar as habilidades afetadas e do uso de estratégias de compensação. Intervenções voltadas para as dificuldades de funções executivas incluem o planejamento antecipado de ações, decomposição de planos em etapas ou "passo a passo" e checklist de tarefas, como formas de organizar e monitorar o comportamento (Sohlberg, 2009; von Cramon, Matthes-von Cramon, \& Mai, 1991).

Tradicionalmente, na área de Neuropsicologia existem muitos estudos de avaliação das funções cognitivas, devido aos esforços para se ampliar a compreensão do funcionamento cognitivo, tanto de pessoas saudáveis, quanto de indivíduos que sofreram algum tipo de alteração ou disfunção cerebral. Dados quantitativos advindos de instrumentos padronizados, facilitam a comparação entre testes e grupos de indivíduos. Por outro lado, estudos de reabilitação neuropsicológica apresentam um desafio metodológico maior, principalmente pela neces- sidade frequente de se individualizar estratégias focadas em alterações funcionais e não somente em treino de função especificamente. Isto porque o objetivo maior do tratamento é o ganho de autonomia nas atividades diárias (Battistella \& Brito, 2002; Organização Mundial da Saúde [OMS], 1980), o que muitas vezes só pode ser atingido através de adaptações e compensações de dificuldades. Neste sentido, estudos de caso em reabilitação neuropsicológica de lesões adquiridas são uma fonte importante de descrições e modelos práticos de atuação com pacientes e seus familiares, pois trazem informações do contexto clínico e obstáculos comuns ao dia a dia de quem lida com estes casos (Bolognani, Gouveia, Brucki, \& Bueno, 2000; Fish, Manly, \& Wilson, 2008; Fleming, Shum, Strong, \& Lightbody, 2005; Gouveia, Brucki, Bolognani, Bezerra, \& Bueno, 2000; Gouveia, Prade, Lacerda, Boschetti, \& Andreoli, 2009; Wall, Turner, \& Clarke, 2013).

O objetivo deste estudo é apresentar um relato de caso de sintomas de desconexão inter-hemisférica, pouco usuais, acompanhado de alterações de funções executivas após lesão encefálica adquirida. Espera-se ainda apresentar estratégias de reabilitação neuropsicológica e avaliar sua aplicabilidade neste caso. Este estudo foi aprovado pelo Comitê de Ética em Pesquisa do Hospital Israelita Albert Einstein (CAAE: 26623814.8.0000.0071).

\section{Relato de Caso}

Paciente D.T., sexo masculino, 33 anos, canhoto, casado (sua esposa estava grávida do primeiro filho do casal), formação superior em Ciências contábeis, com pós-graduação. Atuava como gerente tributário em empresa privada. Em maio de 2011 passou a apresentar alterações visuais e seu oftalmologista o encaminhou ao neurologista, que diagnosticou adenoma hipofisário, após exames. O paciente foi então submetido a procedimento cirúrgico para ressecção do tumor. Foi realizada craniotomia fronto-têmporo-parietal esquerda e no pós-operatório o paciente evoluiu com bacteremia, embolia pulmonar e trombose em membro inferior esquerdo. 
Após a alta, apresentava queixas de dificuldades de concentração em atividades prolongadas, apatia, dificuldades de escrita, para digitar no computador e realizar atividades com a mão esquerda. Demonstrava ainda ansiedade, tristeza e irritabilidade. O exame de Ressonância Magnética (RM) de cabeça realizado em junho do mesmo ano apontou para sinais de manipulação cirúrgica da sela túrcica, com alterações pós-operatórias nas partes moles, junto da craniotomia fronto-têmporo-parietal esquerda, alteração de sinal no corpo caloso, giro do cíngulo, substância branca do lobo frontal e cabeça do núcleo caudado à esquerda, compatível com evento isquêmico em fase subaguda, no território de ramos da artéria cerebral anterior esquerda.

O paciente procurou Centro de Reabilitação do Hospital Albert Einstein e iniciou acompanhamento com médico fisiatra. Ele foi encaminhado para reabilitação multiprofissional, com sessões de fisioterapia, terapia ocupacional, fonoaudiologia e neuropsicologia.

\section{Avaliação Neuropsicológica}

A primeira avaliação, realizada dois meses após a cirurgia, indicou dificuldades discretas quanto às habilidades de memória operacional, amplitude de aprendizagem verbal, fluência verbal e funções visuais (identificação, síntese e organização perceptual). Também foram observadas dificuldades moderadas a graves relacionadas à atenção/funções executivas, funções motoras (grafo-motora, escrita e manipulação bimanual simultânea), habilidades viso-espaciais (praxia construtiva simples e complexa) e de velocidade de processamento de informações (Tabela 1).

O paciente apresentou ainda alterações associadas a disfunções frontais e à síndrome de desconexão hemisférica. Desta forma, em atividades grafo-motoras envolvendo escrita e produção de desenhos, exibiu dificuldades graves em relação à escrita, com evidente competição entre as mãos pelo uso do lápis. $\mathrm{O}$ mesmo comportamento competitivo entre as mãos foi observado sempre que era solicitado a manipular objetos e realizar atividades que demandassem uso simultâneo das mãos. Em atividades de construção de desenhos com cubos, por exemplo, por diversas vezes a mão direita (auxiliada pela linguagem) colocava as peças na posição correta para formar o desenho requerido, enquanto a mão esquerda retirava a peça do lugar, colocando-a em posição errada. Em outra tarefa de abstração viso-espacial, era a mão esquerda que apontava a resposta correta, enquanto a mão direita apontava a erra$\mathrm{da}$, condizente com a resposta verbal igualmente equivocada (hemisfério esquerdo).

Em tarefa de avaliação de agnosia tátil, o paciente demonstrou dificuldades graves no reconhecimento e nomeação de objetos sem apoio visual, ao usar a mão esquerda. Contudo, quando esta mesma atividade foi realizada com a possibilidade de múltipla escolha de resposta (com presença dos objetos), o paciente foi capaz de identificar prontamente e nomear cada elemento, devido à presença de informação visual para ambos os hemisférios.

Os resultados da avaliação neuropsicológicas do paciente estão na Tabela 1 . Devido à alta escolaridade e nível cultural prévio, seus resultados na avaliação foram comparados com normas de população semelhante. D.T. foi reavaliado 5 meses após a primeira avaliação (7 meses após a lesão) e apresentou melhora na maioria das habilidades examinadas. Na reavaliação foi utilizado o método de comparação entre 2 avaliações proposto por Strauss, Sherman, e Spreen (2006), no qual é calculado o Intervalo de Confiança do índice de Mudança Confiável para os escores da primeira avaliação. Deste modo, para cada escore da primeira avaliação é atribuído um intervalo de confiança e, caso os escores da segunda avaliação estejam fora deste intervalo, considera-se que houve mudança significativa entre os escores da primeira e segunda avaliação. Neste estudo optamos por um intervalo de confiança de $90 \%$.

\section{Reabilitação Neuropsicológica}

O tratamento se iniciou seis semanas após a avaliação neuropsicológica. Ao final da avaliação o paciente foi orientado sobre os sintomas decorrentes da desconexão hemisférica, que causavam estranheza e perplexidade ao paciente e sua família. Ele não entendia por- 
quê sua mão (especialmente a esquerda) não lhe obedecia e porquê suas mãos pareciam agir de forma independente. Após perceber e compreender melhor os sintomas de concorrência manual, o paciente foi orientado a tentar realizar as atividades manuais com apenas uma das mãos. Assim, D.T. colocava uma das mãos para trás e realizava a atividade com a outra, quando possível, diminuindo a concorrência entre elas. No entanto, com o passar dos dias, no início da reabilitação formal, estas queixas haviam diminuído bastante pela própria evolução do quadro neurológico. Em seu funcionamento diário persistia apenas a dificuldade de escrita relacionada à esta condição.

Ao iniciar o programa de reabilitação o paciente estava em uso de sertralina, e estava voltando a dirigir. Apresentava diminuição das queixas de concorrência manual, porém, mantinha falhas de memória para compromissos, tarefas e para tomar medicação, diminuição da iniciativa, mas nutria expectativa de retornar ao trabalho o mais breve possível. Outra queixa relevante, associada às funções executivas, era descrita como "paralisia" ou sensação de "travar" para iniciar uma determinada ação ou para decidir o que fazer. Ex.: chegava à padaria e ficava paralisado, sem decidir se pedia algo para levar ou se sentava para fazer uma refeição. Ou então, surpreendido pela chuva na saída de um local, não sabia como proceder. O paciente realizou o tratamento 2 vezes por semana, por um período de 8 meses, com mais dois meses de follow up com sessões mensais.

As metas propostas foram elencadas juntamente com paciente e familiar, priorizando-se as queixas de maior impacto funcional, como lembrar-se de compromissos e de tomar medicação. Muitas das estratégias introduzidas para lembrar-se de realizar determinadas ações estavam mais associadas às falhas de funções executivas, que de memória em si, pelo fato da avaliação formal ter indicado preservação da memória episódica e desta informação corroborar queixas de memória prospectiva e não de recordação de eventos e dados recentes. As dificuldades de escrita foram abordadas nas sessões de fonoaudiologia. Inicialmente elas incluíam o treino através de letras de forma e de digitação no computador, até que o paciente automatizasse novamente o planejamento motor na produção da escrita. Posteriormente o paciente passou a treinar o uso da letra cursiva, mais difícil para ele.

As metas foram revistas a partir de um evento que mudou toda a sua rotina e representava grande desafio para o paciente: o retorno ao trabalho. Tratava-se de uma situação delicada, que ocorreu um pouco antes do previsto, pois o paciente foi liberado pela perícia antes do período recomendado pelo médico. Além de gerar ansiedade, o retorno trazia o risco do paciente não conseguir retomar seu cargo anterior. Neste sentido, novas estratégias foram criadas, baseadas nas queixas e observações trazidas pelo próprio paciente, que se sentia perdido e estava inseguro sobre como organizar suas tarefas.

Como o paciente foi liberado para retornar ao trabalho prematuramente, havia a possibilidade dele não conseguir executar suas atividades de trabalho e o receio de que seu empregador e sua equipe de trabalho pudessem perceber alguma dificuldade e que esta situação fosse expor negativamente o paciente por suas limitações, ameaçando sua permanência na empresa. Neste caso, o empregador realizou uma entrevista com o paciente antes de seu retorno e não demonstrou preocupação quanto à capacidade do paciente, por entender que ele de fato estaria recuperado.

Inicialmente, foi negociada uma redução de jornada de trabalho, a ser aumentada gradualmente, o que não durou muito tempo, pela necessidade de cumprir várias tarefas e desejo do próprio paciente em não demonstrar dificuldades, embora isto o deixasse muito cansado nas primeiras semanas. Após a inserção de adaptações para as atividades de trabalho, foi realizado contato com um funcionário que trabalhava com ele há bastante tempo, uma vez que sua chefia direta havia mudado. O contato com este colega serviu para levantar possíveis problemas e falhas do paciente no dia a dia, sem expô-lo através de um contato formal com a empresa, questionando seu rendimento.

A seguir serão descritas na Figura 1, as principais intervenções realizadas durante a reabilitação neuropsicológica. 


\section{Metas e Objetivos Estratégias de Intervenção $\quad$ Resultados}

\section{Metas iniciais}

Lembrar-se de tomar - Uso de alarme no celular. remédio no horário.

Não perder

compromissos.

Ampliar atividades na rotina / Melhorar iniciativa.

Melhorar atenção.

Diminuir episódios de desorganização em situações cotidianas ("travar").

Melhorar planejamento e abstração.
- Uso de agenda no celular e da função lembrete com alarme.

- Esposa foi orientada a solicitar mais auxílio em tarefas rotineiras, como fazer compras;

- Organização da rotina com atividades fixas, como exercícios de fonoaudiologia e atividade física; - Introdução de lista de atividades diárias, com atividades selecionadas pelo paciente e esposa.

- Treino através de exercícios de lápis e papel e de computador: Rehacom (Schuhfried, 2003).

- Uso de passo a passo em situações com maior frequência de ocorrências, como ir à padaria, por exemplo; - Orientação para generalizar a estratégia para outras situações.

- Exercícios de planejamento com situações hipotéticas para solução de problemas.

- Exercícios de texto para interpretação de sentido e geração de hipóteses.
Paciente passou a tomar a medicação no horário correto.

Paciente deixou de esquecer a maioria de seus compromissos.

Paciente ampliou quantidade de atividades na rotina, desde compras e auxílio à esposa, como exercícios de fono. Apenas foi resistente à atividade física, que deixou de praticar regularmente.

Diminuição de erros e aumento de velocidade de resposta em exercícios de atenção sustentada, dividida, controle inibitório e memória operacional.

Paciente passou a planejar antes de sair de casa, o que iria pedir na padaria, além de se imaginar ensaiando mentalmente a atividade.

Em situações inesperadas, como deparar-se com chuva ao sair do escritório sem guarda-chuva, tentava parar e pensar nas opções. Eventualmente ainda ficava "paralisado", mas depois reagia.

- Diminuiu tempo de geração de respostas e melhorou a qualidade das mesmas.

- Melhorou a compreensão de sutilezas

e conteúdos mais complexos nos exercícios.

\section{Metas após o retorno ao trabalho}

Ampliar percepção de funções executivas.

Aumentar iniciativa e organização para tarefas no trabalho. das dificuldades

- Psicoeducação através de textos sobre funções executivas e estratégias para manejo de dificuldades;

- Análise de episódios que ocorriam com ele para ilustrar as dificuldades.

- Lista de tarefas no trabalho

(To do List);

- Passo a passo para resolução de problemas.

Voltar a fazer anotações em reuniões de trabalho.

Checar seu desempenho no ambiente de trabalho.
- Uso de Tablet para compensar erros de escrita que o constrangiam em público.

- Contato telefônico com colega de trabalho próximo a D.T. para saber se ele observava problemas cognitivos e ou de comportamento.
Paciente passou a perceber melhor e admitir dificuldades que antes minimizava. A partir dos episódios trazidos era possível elaborar estratégia específica para a situação.

- Deixou de ficar esperando que alguém lhe pedisse para fazer algo. Passou a seguir a lista, evitando que as tarefas atrasassem.

- Diante de demandas novas, passou a elaborar plano de ação para não ficar paralisado, sem saber como começar. Eventualmente ainda ficava inseguro e tinha dificuldade para se organizar.

- Retomou anotações com o uso do recurso eletrônico, sem constrangimento.

- Seu colega não referiu observação de dificuldades na realização das tarefas ou para realizar reuniões com a equipe. Apenas notou paciente mais quieto que anteriormente ao quadro neurológico. 
Ao final da reabilitação, que durou cerca de 8 meses, o paciente estava trabalhando, bem adaptado a sua nova rotina, com o uso das estratégias descritas. Houve diminuição significativa das queixas anteriores e o paciente conseguia ser mais bem sucedido para manejar problemas novos, ou inesperados em casa e no trabalho, utilizando as estratégias aprendidas. Estas envolviam passo-a-passo e prática de auto monitoramento, para replanejar suas ações sempre que percebesse que não sabia como agir.

Sua esposa ainda considerava que sua iniciativa estava um pouco reduzida, tendo por base seu perfil pré-morbido, assim como a pró-atividade para ajudar em algumas situações com a filha pequena e para tomar decisões. No entanto, ela já não se sentia desamparada e sobrecarregada com a administração da casa e da família, da mesma forma que antes. Devido à diminuição da frequência dos episódios de "paralisia" e tomada de decisão, ele se tornou mais apto a assumir tarefas na casa e cuidados com a filha, diminuindo a necessidade de solicitação e supervisão da esposa.

A comparação entre as duas avaliações demonstrou melhora significativa de desempenho em diversos instrumentos, na maioria das habilidades avaliadas, como as habilidades de: memória operacional, atenção dividida, habilidades grafo-motoras (incluindo a remissão de comportamentos de competição manual), memória verbal e visual, praxia construtiva, cálculos, funções executivas e funcionamento intelectual (esferas verbal e de execução). A avaliação do humor também denotou redução dos sintomas depressivos, o que corroborou a observação durante os atendimentos e a percepção de paciente e familiar. Por outro lado, foi possível observar a persistência de alterações discretas de funções executivas, que se manifestavam em situações mais complexas, envolvendo situações novas e tomada de decisões, motivo pelo qual o paciente ainda necessitava utilizar estratégias específicas, especialmente no trabalho.

\section{Discussão}

Neste estudo foi apresentado relato de caso de avaliação e reabilitação de paciente com al- terações cognitivas, envolvendo sintomas de disfunção executiva e síndrome de desconexão inter-hemisférica. O paciente evoluiu com remissão rápida dos sintomas de desconexão, porém necessitou de estratégias para minimizar alterações de atenção e funções executivas, atingindo resultados satisfatórios em termos de adaptação e retorno às atividades prévias. Deve-se ressaltar o papel da melhora espontânea na recuperação do paciente, devido ao curto prazo decorrido da lesão no início do processo, assim como da gravidade da lesão (leve a moderada) (Gouveia, 2006; Kolb, 2004). Neste caso, aliar a condição recente da lesão com treinos de funções cognitivas demonstrou bons resultados. Em geral, pacientes com grau de comprometimento severo e/ ou acometimentos de várias esferas cognitivas e maior tempo de lesão (condição crônica), costumam demonstrar melhora limitada das funções cognitivas propriamente ditas, medidas na avaliação neuropsicológica formal.

As intervenções de reabilitação contribuem de forma mais significativa para o bem estar do paciente, quando priorizam a sua autonomia e funcionalidade, abordando as queixas presentes e não apenas os déficits cognitivos indicados na avaliação formal. (Ben-Yishay, 2000; Gouveia et al., 2009; Trexler, 2000). Muitas das estratégias utilizadas neste caso tinham como objetivo minimizar dificuldades sutis de funções executivas. Apesar do desafio, há uma vantagem muito grande de se desenvolver estratégias personalizadas para a demanda do paciente, ou seja, criar e adaptar recursos para o tipo de situação em que o paciente sente dificuldade. Uma questão relevante, que geralmente ocorre nestes casos de alterações de funções executivas é que o paciente nem sempre percebe suas limitações e tende a minimizar lapsos e falhas, devido a anosognosia (Goldberg, 2002). Neste caso, os relatos da esposa e de um colega de trabalho do paciente auxiliaram na identificação de algumas situações importantes ao longo do processo.

Ao longo do tratamento é preciso acompanhar as situações do dia a dia em que as dificuldades se manifestam, para se propor algo contextualizado e ao mesmo tempo viável para ser executado. Além disto, é sempre importante che- 
Tabela 1

Comparação dos Resultados entre as Duas Avaliações Neuropsicológicas

\begin{tabular}{|c|c|c|c|c|c|c|}
\hline & EB 1 & Perc 1 & EB 2 & Perc 2 & RCI-IC 90\%* & Comparação \\
\hline \multicolumn{7}{|l|}{ Atenção e Funções Executivas } \\
\hline Dígitos Diretos & 6 & 31 & 6 & 31 & 5,06 a 6,94 & ns \\
\hline Dígitos Inversos & 4 & 27 & 4 & 27 & 4,00 a 5,00 & ns \\
\hline Teste de Trilhas A & 157 & 0,1 & 48 & 4 & 145,95 a 168,05 & $\uparrow$ \\
\hline Teste de Trilhas B & 431 & 0,1 & 69 & 42 & 413,00 a 449,00 & $\uparrow$ \\
\hline Stroop 1 & 11 & 54 & 9 & 90 & 9,77 a 12,23 & $\uparrow$ \\
\hline Stroop 2 & 11 & 86 & 12 & 76 & 8,46 a 13,54 & ns \\
\hline Stroop 3 & 15 & 86 & 14 & 88 & 8,38 a 21,62 & ns \\
\hline WCST Categorias & 1 & 0,6 & 2 & 4 & 0 a 2,83 & ns \\
\hline WSCT Erros & 28 & 2 & 19 & 18 & 18,13 a 37,87 & ns \\
\hline WCST Resp. Persev. & 8 & 34 & 15 & 1 & 1,62 a 14,38 & $\downarrow$ \\
\hline WCST Erros Persev. & 7 & 34 & 13 & 0,7 & 2,16 a 11,84 & $\downarrow$ \\
\hline WCST Erros Não Persev. & 21 & 0,7 & 6 & 50 & 13,32 a 28,68 & $\uparrow$ \\
\hline WCST Perda de Meta & 1 & 18 & 1 & 18 & 0 a 2,17 & ns \\
\hline \multicolumn{7}{|l|}{ Memória } \\
\hline RAVLT A1 & 4 & 8 & 6 & 42 & 2,75 a 5,25 & $\uparrow$ \\
\hline RAVLT A5 & 12 & 58 & 14 & 84 & 10,17 a 13,83 & $\uparrow$ \\
\hline RAVLT Total & 40 & 21 & 58 & 82 & 32,45 a 47,55 & $\uparrow$ \\
\hline RAVLT A6 & 7 & 21 & 11 & 66 & 4,58 a 9,42 & $\uparrow$ \\
\hline RAVLT A7 & 7 & 18 & 10 & 50 & 7,17 a 10,83 & $\uparrow$ \\
\hline Memória Lógica I (WMS-R) & 29 & 62 & 34 & 90 & 19,83 a 38,17 & ns \\
\hline Memória Lógica II (WMS-R) & 20 & 41 & 29 & 80 & 10,06 a 29,94 & ns \\
\hline Reprodução Visual I (WMS-R) & 18 & 2 & 38 & 90 & 10,67 a 25,33 & $\uparrow$ \\
\hline Reprodução Visual II (WMS-R) & 6 & 1 & 40 & 99 & 0,00 a 14,43 & $\uparrow$ \\
\hline \multicolumn{7}{|l|}{ Linguagem } \\
\hline Fluência Verbal Fonêmica & 41 & 38 & 46 & 54 & 30,29 a 51,71 & ns \\
\hline Fluência Verbal Semântica & 16 & 14 & 28 & 86 & 10,84 a 21,16 & $\uparrow$ \\
\hline Nomeação de Boston & 56 & 54 & 57 & 62 & 53,36 a 58,64 & ns \\
\hline \multicolumn{7}{|l|}{ Habilidade Visoespaciais } \\
\hline Desenho do Relógio & 12 & 0,1 & 13 & 0,3 & 11,35 a 12,65 & $\uparrow$ \\
\hline Hooper V O T & 29 & 76 & 30 & 82 & 26,07 a 30,00 & ns \\
\hline TFC Rey - Cópia & 24 & 0,3 & 33 & 54 & 19,32 a 28,68 & $\uparrow$ \\
\hline TFC Rey - Memória & 22 & 69 & 29 & 95 & 15,11 a 28,89 & $\uparrow$ \\
\hline
\end{tabular}

IDD 2 Perc. 1 IDD 2 Perc. 2 RCI-IC 90\%* Comparação

Funções Intelectuais

$\begin{array}{lcccccc}\text { WAIS-III - Vocabulário } & 10 & 50 & 9 & 37 & 8,30 \text { a } 11,70 & \text { ns } \\ \text { WAIS-III - Semelhanças } & 9 & 37 & 11 & 63 & 6,83 \text { a } 11,17 & \text { ns } \\ \text { WAIS-III - Aritmética } & 9 & 37 & 11 & 63 & 6,32 \text { a } 11,68 & \text { ns }\end{array}$




\begin{tabular}{lcccccc} 
WAIS-III - Dígitos & 7 & 16 & 8 & 25 & 4,42 a 9,58 & ns \\
WAIS-III - Informação & 10 & 50 & 12 & 75 & 8,41 a 11,59 & $\uparrow$ \\
WAIS-III - Seq. Núm-Letras & 5 & 5 & 9 & 37 & 1,85 a 8,15 & $\uparrow$ \\
WAIS-III - Comp. Figuras & 6 & 9 & 9 & 37 & 2,81 a 9,19 & ns \\
WAIS-III - Códigos & 2 & 0,4 & 9 & 2 & 0 a 4,69 & ns \\
WAIS-III - Cubos & 6 & 9 & 9 & 37 & 3,83 a 8,17 & $\uparrow$ \\
WAIS-III - Rac. Matricial & 9 & 37 & 11 & 63 & 5,98 a 12,02 & ns \\
WAIS-III - Proc. Símbolos & 4 & 2 & 9 & 37 & 1,15 a 6,85 & $\uparrow$ \\
\hline & QI 1 & Perc. 1 & QI 2 & Perc. 2 & RCI-IC $90 \% *$ & Comparação \\
\hline Índices de QI & & & & & & $\uparrow$ \\
WAIS-III - QI Verbal & 93 & 32 & 102 & 55 & 86,32 a 99,68 & $\uparrow$ \\
WAIS-III - QI Execução & 74 & 4 & 87 & 19 & 63,88 a 84,12 & $\uparrow$ \\
WAIS-III - QI Total & 84 & 14 & 96 & 39 & 77,37 a 90,63 & $\uparrow$ \\
WAIS-III - Comp. Verbal & 98 & 45 & 103 & 58 & 90,69 a 105,31 & ns \\
WAIS-III - Org. Perceptual & 82 & 12 & 97 & 42 & 70,51 a 93,49 & $\uparrow$ \\
WAIS-III - Memória Operac. & 82 & 12 & 95 & 37 & 70,85 a 93,15 & $\uparrow$ \\
WAIS-III - Vel de Process. & 66 & 1 & 81 & 10 & 54,67 a 77,33 & $\uparrow$ \\
\hline
\end{tabular}

Notas. Legenda: $\mathrm{ns}=$ Mudança de desempenho não significativa; $\uparrow=$ Melhora significativa de desempenho; $\downarrow=$ Piora significativa de desempenho; EB1= Escore Bruto da primeira avaliação; EB2= Escore Bruto da segunda avaliação; Perc. 1= Percentil da primeira avaliação; Perc. 2= Percentil da segunda avaliação; Teste das Trilhas A e B (Campanholo et al., 2014; Reitan, 1958); Stroop=Teste de Cor Palavra de Stroop (Campanholo et al., 2014; Strauss et al., 2006); WCST= Teste de Classificação de Cartas de Wisconsin (Heaton, Chelune, Talley, Kay, \& Curtiss, 1993); RAVLT= Teste de Aprendizagem Auditivo Verbal de Rey (Lacerda, Andreoli, \& Andreoli, 2008; Schmidt, 1996); WMS-R= Escala Wechsler de Memória Revisada (Martins, Bolognani, Pompeia, Bueno, \& Miranda, 2015; Wechsler, 1987); Hooper VOT= Teste de Organização Visual de Hooper (Strauss et al., 2006; Tosello, 2005); TFC= Rey -Teste da Figura Complexa de Rey (Osterrieth, 1944; Strauss et al., 2006); WAIS-III= Escala Wechsler de Inteligência para Adultos Terceira Edição (Wechsler, 2004); Seq. Núm-Letras= Sequencia Números e Letras; Comp. Figuras $=$ Completar Figuras; Rac. Matricial $=$ Raciocínio Matricial; Proc. Símbolos= Procurar Símbolos; QI= Coeficiente Intelectual; Comp. Verbal= Compreensão Verbal; Org. Perceptual= Organização Perceptual; Memória Operac.= Memória Operacional; Vel. de Process. $=$ Velocidade de Processamento. $*$ RCI-IC $=$ Intervalo de Confiança do Índice de Mudança Confiável: RCI-CI= EB1 $\pm\left(z .\left(\sqrt{ } 2 .(D P . \sqrt{ } 1-\alpha)^{2}\right)\right.$, onde, $z$ é valor do intervalo de confiança, $D P$ é o desvio padrão da amostra de normatização e $\alpha$ é o valor de confiabilidade teste-reteste (Strauss et al., 2006).

car a efetividade do auxílio e adaptá-lo, se for necessário (Wilson, 1999). Um exemplo disto foi o retorno ao trabalho. Novas metas foram criadas a partir desta mudança, para abarcar situações importantes e que envolviam demandas cognitivas que não estavam presentes anteriormente. O retorno ao trabalho é uma etapa bastante delicada e nem sempre possível para pacientes com limitações graves, que necessitarão de mudanças significativas em suas demandas de trabalho, em termos de quantidade de trabalho, tempo para sua execução, complexidade e responsabilidade envolvida no tipo de tarefa. Neste caso, pode-se negociar uma mudança de área ou local de trabalho, quando existe esta possibilidade. Pacientes que trabalham em empresas familiares ou que contam com maior flexibilidade de tempo e ambiente para desempenhar suas tarefas, em geral conseguem retornar com mais facilidade e menor pressão. Até pelo fato de que podem mudar mais facilmente de atividade.

Sempre que o retorno ao trabalho for considerado, é preciso estudar quais as atividades e funções a serem exercidas, para se fazer um levantamento das demandas cognitivas envolvidas, bem como dos fatores ambientais que podem favorecer, ou dificultar o desempenho do paciente. Um exemplo disto seria um ambiente com distratores, muitas pessoas, barulho. Como estes pacientes costumam apresentar dificul- 
dades de supervisão atencional e organização, beneficiam-se de ambientes fechados e rotina de atividades estruturada. O levantamento destas informações permite uma análise de cada etapa, para que sejam elaborados treinos específicos e realizadas adaptações para minimizar condições desfavoráveis. Na prática diária, observamos com frequência que o público em geral não tem conhecimento do tipo de alterações cognitivas ou comportamentais, que os indivíduos com lesão encefálica adquirida podem apresentar. Se por um lado, isto poderia diminuir o estigma do paciente que tenta retomar suas atividades profissionais após uma lesão cerebral, por outro, dificulta a aceitação e a compreensão da necessidade de se propor adaptações e modificações no ambiente de trabalho. Quando o indivíduo demonstra alguma dificuldade, ao invés de isto ser encarado como algo específico, relacionado a uma habilidade, observa-se uma série de confusões por parte de colegas e chefes, que atribuem as dificuldades a problemas de humor, de motivação e engajamento, o que acaba deteriorando o relacionamento do paciente no ambiente de trabalho. Diante deste cenário, faz-se necessário orientar as pessoas de seu relacionamento direto quanto às mudanças ocorridas com o paciente, $o$ motivo pelo qual se comporta de forma distinta e suas dificuldades atuais, visando ampliar a tolerância e a aceitação das adaptações, necessárias para que o paciente volte a se inserir na rotina do setor. Desta forma, o retorno ao trabalho é uma meta ambiciosa durante o processo de reabilitação, que deve ser avaliada com cautela, pois é preciso evitar que o indivíduo se coloque em uma situação desfavorável, expondo suas limitações, ao mesmo tempo em que ele precisa de compreensão e suporte no ambiente de trabalho, para fazer uso de adaptações e se adequar às demandas de sua função (Bjorkdahl, 2010; Hofgren, Esbjornsson, \& Sunnerhagen, 2010; Hooson, Coetzer, Stew, \& Moore, 2013).

Cabe ressaltar que, o atual estudo apresenta algumas limitações importantes, como por exemplo, o papel da recuperação espontânea durante o tratamento de reabilitação, uma vez que o paciente ainda estava em fase recente de recuperação da lesão adquirida. Outro ponto a ser considerado, foi a ausência de intervenções específicas para os sintomas de desconexão hemisférica, que remitiram quase totalmente de forma espontânea, especialmente pelo fato do paciente demorar algumas semanas para iniciar as sessões de reabilitação neuropsicológica, após finalizar a avaliação das funções cognitivas.

Estudos de metanálise em reabilitação neuropsicológica criticam o baixo número de trabalhos prospectivos, controlados e randomizados nesta área (Cicerone et al., 2011), o que dificulta o consenso sobre as melhores práticas de intervenção, especialmente no que se refere ao treino de funções cognitivas. Por outro lado, alguns estudos sugerem que o uso de estratégias compensatórias e treinos personalizados para as dificuldades dos pacientes, permite que as metas sejam avaliadas de acordo com os ganhos em funcionalidade e autonomia que elas proporcionam, resultando em boas evidências (Bahar-Fuchs, Clare, \& Woods, 2013). Quando consideramos o uso de estratégias compensatórias, os relatos de caso representam uma fonte importante de informações, pois exemplificam as formas de inserção dos auxílios na rotina do paciente e descrevem formas de lidar com falhas e adaptações individualizadas dos recursos utilizados. Esperamos que este estudo tenha apresentado de forma clara as metas e as etapas do trabalho de reabilitação, no manejo das dificuldades cognitivas ora descritas. Estudos futuros poderiam abordar a questão específica do retorno ao trabalho, pois há poucos trabalhos na literatura que apresentem a inserção destes pacientes em atividades produtivas, seja buscando um novo cargo ou tentando retomar sua posição anterior.

\section{Referências}

Bahar-Fuchs, A., Clare, L., \& Woods, B. (2013). Cognitive training and cognitive rehabilitation for persons with mild to moderate dementia of the Alzheimer's or vascular type: A review. Alzheimer's Research \& Therapy, 5(4), 35. doi:10.1186/alzrt189

Battistella, L. R., \& Brito, C. M. M. (2002). Classificação internacional da funcionalidade. Acta Fisiátrica, 9, 98-101. 
Ben-Yishay, Y. (2000). Neuropsychological rehabilitation: A holistic perspective. In A.-L. Christensen \& B. P. Uzzell (Eds.), International Handbook of Neuropsychological Rehabilitation. New York: Kluwer Academic.

Bjorkdahl, A. (2010). The return to work after a neuropsychological programme and prognostic factors for success. Brain Injury, 24(9), 1061-1069. doi:10.3109/02699052.2010.494588

Bolognani, S. A., Gouveia, P. A., Brucki, S. M., \& Bueno, O. F. (2000). [Implicit memory and its contribution to the rehabilitation of an amnesic patient: Case report]. Arquivos de NeuroPsiquiatria, 58(3B), 924-930.

Browndyke, J. N. (2002). Callosal disconnexion syndromes. Neuropsychology Central. Retrieved from http://www.neuropsychologycentral.com/

Campanholo, K. R., Romão, M. A., Machado, M. A. R., Serrao, V. T., Coutinho, D. G. C., Benute, G. R. G., ...Lucia, M. C. S. (2014). Performance of an adult Brazilian sample on the Trail Making Test and Stroop Test. Dementia e Neuropsychologia, 8(1), 26-31.

Cicerone, K. D., Langenbahn, D. M., Braden, C., Malec, J. F., Kalmar, K., Fraas, M., ...Ashman, T. (2011). Evidence-based cognitive rehabilitation: Updated review of the literature from 2003 through 2008. Archives of Physical Medicine and Rehabilitation, 92(4), 519-530. doi:10.1016/j.apmr.2010.11.015

Fish, J., Manly, T., \& Wilson, B. A. (2008). Long-term compensatory treatment of organizational deficits in a patient with bilateral frontal lobe damage. Journal of the International Neuropsychological Society, 14(1), 154-163. doi:10.1017/S1355617708080120

Fleming, J. M., Shum, D., Strong, J., \& Lightbody, S. (2005). Prospective memory rehabilitation for adults with traumatic brain injury: A compensatory training programme. Brain Injury, 19(1), $1-10$.

Geschwind, N. (1965a). Disconnexion syndromes in animals and man. I. Brain, 88(2), 237-294.

Geschwind, N. (1965b). Disconnexion syndromes in animals and man. II. Brain, 88(3), 585-644.

Gil, R. (2002). Neuropsicologia. São Paulo, SP: Santos.

Goldberg, E. (2002). O cérebro executivo: Lobos frontais e a mente civilizada. Rio de Janeiro, RJ: Imago.
Gouveia, P. A. R. (2006). Introdução à reabilitação neuropsicológica de adultos. In J. Abrisqueta-Gomes \& F. H. Santos (Eds.), Reabilitação Neuropsicológica da teoria à prática (pp. 7382). São Paulo, SP: Artes Médicas.

Gouveia, P. A. R., Brucki, S. M., Bolognani, S. A., Bezerra, A. B., \& Bueno, O. F. (2000). [Procedures to improve daily activities performance in individuals with cerebral anoxia: Case report]. Arquivos de Neuro-Psiquiatria, 58(2B), 560-565.

Gouveia, P. A. R., Prade, C. V., Lacerda, S. S., Boschetti, W. L., \& Andreoli, P. B. (2009). Reabilitação neuropsicológica em fase aguda e crônica após Traumatismo Crânio-Encefálico (TCE) grave: Relato de caso. Contextos Clínicos, 2(1), 18-26.

Heaton, R. K., Chelune, G. J., Talley, J. L., Kay, G. C., \& Curtiss, G. (1993). Wisconsin Card Sorting Test Manual. Revised and Expanded. Odessa, FL: Psychological Assessment Resources.

Hofgren, C., Esbjornsson, E., \& Sunnerhagen, K. S. (2010). Return to work after acquired brain injury: Facilitators and hindrances observed in a sub-acute rehabilitation setting. Work, 36(4), 431-439. doi:10.3233/WOR-2010-1039

Hooson, J. M., Coetzer, R., Stew, G., \& Moore, A. (2013). Patients' experience of return to work rehabilitation following traumatic brain injury: A phenomenological study. Neuropsychological Rehabilitation, 23(1), 19-44. doi:10.1080/09602 011.2012 .713314

Kolb, B. (2004). Mechanisms of Cortical Plasticity after Neuronal Injury. In J. Ponsford (Ed.), Cognitive and Behavioral Rehabilitation: From neurobiology to clinical practice. New York: The Guilford Press.

Lacerda, S. S., Andreoli, P. B., \& Andreoli, S. B. (2008). Reliability of the Brazilian version of Rey auditory and verbal learning test (RAVLT-BR). Journal of the International Neuropsychological Society, 14(Suppl. 2), 9-9.

Levine, B., Schweizer, T. A., O'Connor, C., Turner, G., Gillingham, S., Stuss, D. T., ...Robertson, I. H. (2011). Rehabilitation of executive functioning in patients with frontal lobe brain damage with goal management training. Frontiers in Human Neuroscience, 5, 9. doi:10.3389/ fnhum.2011.00009

Loring, D. W. (1999). INS Dictionary of Neuropsychology. New York: Oxford University Press. 
Martins, M. R., Bolognani, S. A. P., Pompeia, S., Bueno, O. F. A., \& Miranda, M. C. (2015). Versões alternativas do Subteste Memória Lógica da WMS-R: Análise de desempenho de uma amostra saudável da cidade de São Paulo. Psicologia: Reflexão e Crítica, 28(3), 444-453. doi:10.1590/1678-7153.201528303

Organização Mundial da Saúde. (1980). International Classification of Impairments, Disabilities and Handicaps: A Manual of Classification Relating to the Consequences of Disease. Geneva: Author.

Osterrieth, P. A. (1944). Filetest de copie d'une figure complex: contribution a létude de la perception et de la memoire. Archives de Psychologie, 30, 286-356.

Reitan, R. M. (1958). Validity of the Trail Making Test as an indicator of organic brain damage. Perceptual and Motor Skills, 8, 271-276.

Rubens, A. B., Geschwind, N., Mahowald, M. W., \& Mastri, A. (1977). Posttraumatic cerebral hemispheric disconnection syndrome. Archives of Neurology, 34(12), 750-755.

Schmidt, M. (1996). Rey auditory and verbal learnig test: A handbook. Los Angeles, CA: WPS.

Schuhfried, G. (2003). RehaCom Version 5. Basic Manual [Software]. Retrieved from http://www. hasomed.de/fileadmin/user_upload/RehaCom/ Manuale/ENG/RehaComEN.pdf

Sohlberg, M. M. M. (2009). Reabilitação Cognitiva: Uma Abordagem Neuropsicológica Integrativa. São Paulo, SP: Santos.

Sperry, R. S., Gazzaniga, M. S., \& Bogen, J. E. (1969). Inter-hemispheric relationships: The neocortical commissures; sindromes of hemisferic disconnection. In P. J. Vinken \& Bruyn (Eds.), Handbook of clinical neurology (Vol. 4, pp. 273-290). Amsterdan: North-Holland.

Strauss, E., Sherman, E. M. S., \& Spreen, O. (2006). A compendium of neuropsychological tests: Administration, norms, and commentary ( $3^{\text {rd }}$ ed.). Oxford, UK: Oxford University Press.
Tosello, D. J. T. (2005). Contribuição para o estudo normativo do Hooper Visual Organization Test (VOT). Psicologia Hospitalar, 13(2), 59-83.

Trexler, L. E. (2000). Empirical support for Neuropsychological Rehabilitation. In A.-L. Chistensen \& B. P. Uzzell (Eds.), International Handbook of Neuropsychological Rehabilitation. New York: Kluwer Academic.

Von Cramon, D., Matthes-von Cramon, D., \& Mai, N. (1991). Problem-solving deficits in brain injured patients: A therapeutic approach. Neuropsychological Rehebilitation, 1, 45-64.

Wall, G., Turner, A., \& Clarke, R. (2013). Evaluation of neuropsychological rehabilitation following severe traumatic brain injury: A case report. Neurocase, 19(6), 530-541. doi:10.1080/13554 794.2012 .701642

Wechsler, D. (1987). Wechsler Memory Scale - Revised Edition. Manual. New York: The Psychological Corporation.

Wechsler, D. (2004). Escala de Inteligência Wechsler para Adultos: Manual (3. ed.). São Paulo, SP: Casa do Psicólogo.

Wilson, B. A. (1999). Case Studies in Neuropsychological Rehabilitation. New York: Oxford University Press.

Wilson, B. A. (2008). Neuropsychological rehabilitation. Annual Review of Clinical Psychology, 4, 141-162. doi:10.1146/annurev.clinpsy.4.022007.141212
Recebido: 16/03/2016

$1^{a}$ revisão: $21 / 07 / 2016$ Aceite final: 1\% $1 \% / 2016$ 